\title{
An Empirical Research on Hedging with T-bond Futures in China Xiaoting $\mathrm{Xu}^{1, \mathrm{a}}$,Jianfeng Liang ${ }^{1, \mathrm{~b}^{*}}$ \\ ${ }^{1}$ Lingnan (University) College, Sun Yat-sen University, Guangzhou, China \\ axuxiaot@mail2.sysu.edu.cn, bjfliang@mail.sysu.edu.cn \\ ${ }^{*}$ Corresponding author
}

\begin{abstract}
This paper investigates hedge investment by using T-bond futures in terms of static and dynamic frameworks to control the interest risk of different types of bondsportfolios. The empirical test shows that the hedge performanceare affected by the correlation between the prices of bonds and treasury futures, the length of the bond remaining term and also the credit risk. Specifically, the CTD bond is always with the best performance by hedging with T-bond futures, and other long term deliverable bonds and financial bonds are also well hedged. While the hedging performance to those short term deliverable bonds are not so good,neither to credit bonds.
\end{abstract}

Keywords: T-bond futures, Hedge, Hedge performance evaluation

\section{中国国债期货套期保值实证研究}

\author{
徐小婷 ${ }^{\mathrm{a}}$, 梁建峰 $\mathrm{b}^{*}$ \\ 中山大学岭南 (大学) 学院, 广州, 中国 \\ axuxiaot@mail2.sysu.edu.cn, bjfliang@mail.sysu.edu.cn \\ 通讯作者
}

中文摘要：本文实证研究中国国债期货的套期保值

应用方法和绩效, 具体采用了基点价值法、久期法

以及收益率调整法等对多品种债券进行静态和动态

套期保值, 比较套期保值在不同方法, 不同债券品

种上的绩效表现。实证研究表明, 债券的套期保值

绩效与被套保债券价格、剩余期限长短及信用风险

等性质密切相关。国债期货对最廉交割券的套保效

果最好, 对剩余期限较短的国债和信用债对冲效果

欠佳。

关键词:国债期货；套期保值；套保绩效评价

\section{1. 引言}

我国债券市场在过去十年间得到迅猛 发展, 银行间债券交易量增长超过6倍。2005 年至 2015 年间，人民币债券托管总量从 5 万 多亿元增长到约 29 万亿元; 作为重要的债券 品种, 人民币国债托管总量也从 $2.4 万$ 亿元 增长到 8.5 万亿元。随着金融市场的发展和 利率市场化的加速推进, 我国国债收益率的 波动性也逐步提高, 目前已处于全球较高水 平, 利率风险管理的需要越来越高。中国国 债期货市场应运而生，2013年9月6日，国债 期货正式在中国金融期货交易所上市交易 
并得到迅速发展, 套期保值对冲利率风险也 成为对国债期货的重要投资需求。

期货套期保值研究主要包含两个角度。 一类是效用最大化, 综合考虑资产收益及方 差, 研究均值-风险套保比率。Johnson(1960) 和Stein(1961)引入马科维茨组合投资理论 体系, 提出收益方差最小化的套保率计算公 式为开端。Ederington(1979)将其引入金融 期货, 并给出套保有效性的衡量标准。另外 一类从风险最小化的角度, 对最小风险套保 率进行研究。Witt(1987)利用传统的OLS回 归模型, 对期货市场的套保比率进行估计。 Ghosh(1993)根据Granger和Engle的协整理 论, 提出了ECM模型。国内关于期货套期 保值的研究很多, 但针对国债期货套期保值 的实证研究较少, 更多集中在商品期货和股 指期货。卢太平和刘心报 (2011) 实证研究 了中国股指期货套期保值。程宇和吕广仁 (2010) 研究了利率期货套期保值方法。傅 晓云 (2013) 则讨论了国债期货在商业银行 利率风险管理中的应用。

本文采用中国国债期货上市交易数据 针对多品种债券进行套期保值对比研究, 在 静态套保中采用业内常用的修正久期法和 基点价值法。本文还采用了定期调整套保比 例的方法进行了动态套保研究, 并比较了套 保绩效。

\section{2. 研究方法与数据说明}

国债期货和其他期货产品用于套期保 值类似, 都需要严格的流程控制。国债期货 套保的主要步骤包括, 风险评估, 保值的程 度, 套保方向, 期限, 合约选择, 开仓时机, 套保数量计算, 保证金管理及风险控制, 套 保效果评估等，具体见图1所示。

本文主要研究国债期货套期保值的最 优套期率和套保绩效评价两个环节。在套期 保值的应用上还可以分别进行静态套保和 动态调整, 并进一步比较其套期绩效的改进 效果。

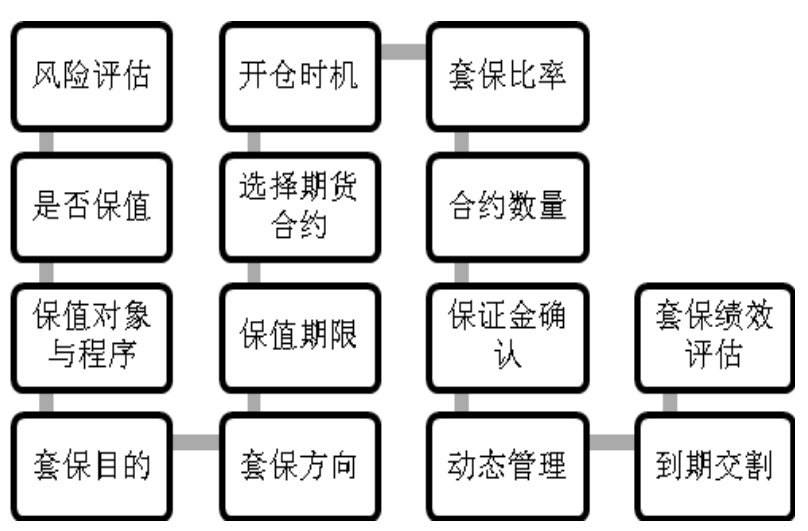

图1 国债期货套期保值流程

\section{1 最优套期率}

（1）基点价值法（BPV）法

基点价值（Basis Point Value, BPV) 是 收益率变动一个基点所导致的资产价格变 动的数量。债券的基点价值可利用久期进行 近似计算:

$$
B P V_{t}=S_{t} * D * \Delta y
$$

其中 $S_{t}$ 是债券当前的价格, $D$ 是债券的 久期, $\Delta y$ 是收益率的变动幅度。

由于债券组合和期货合约价值的变化 可以通过基点价值来体现，通过寻找一个套 保比例, 使得套保组合的基点价值为 0 , 这 个比率就是最优基点价值法套保比率。设被 套保的价格为 $S_{t}$, 基点价值是 $B P V_{t}$ 。国债 期货的价格和基点价值分别为 $S_{f}, B P V_{f}$, 最廉价交割券 $\mathrm{CTD}$ 券的转换因子、价格和基 点价值为 $C F_{C T D}, S_{C T D}, B P V_{C T D}$ 。假设国债收 益率曲线平行移动, 则被套保国债与国债期 货的收益率变化幅度相同, 即需要找到最优 套期率 $H R$ :

$$
\begin{aligned}
& B P V_{t}-H R^{*} B P V_{f}=0 \\
& \Rightarrow H R=B P V_{t} / B P V_{f} \\
& \Rightarrow H R \approx C F_{C T D} * B P V_{t} / B P V_{C T D}
\end{aligned}
$$

（2）修正久期（MD）法

修正久期是衡量债券价格相对于到期 收益率变动敏感性的重要指标, 也可以用与 进一步计算利率期货套期保值比率。 


$$
\begin{aligned}
H R & =B P V_{t} / B V P_{f}=C F_{C T D} * B P V_{t} / B P V_{C T D} \\
& =C F_{C T D} *\left(S_{t} * D_{t}\right) /\left(S_{C T D} * D_{C T D}\right) \\
& =\left(S_{t} * D_{t}\right) /\left(F_{t} * D_{C T D}\right)
\end{aligned}
$$

（3）益率曲线调整法（ $\beta$ 法）

基点价值法和修正久期法中有一个基 本假设, 收益率曲线仅作平移变动。现实中 收益率曲线并不总是平行升降, 而是需要利 用收益率 $\beta$ 值进行修正, $\beta$ 衡量了历史数据 中CTD券收益率变动一个基点, 待套保债券 收益率所变动的幅度。即:

$$
y=\alpha+\beta^{*} y_{C T D}+\varepsilon
$$

因此, 经过 $\beta$ 调整的最优套保率为:

$$
\begin{aligned}
H R_{\beta} & =\beta^{*} H R \\
& \approx \beta^{*} B V P_{t} * C F_{C T D} / B P V_{C T D}
\end{aligned}
$$

静态套保是基于某日的市场情况计算 出套保比例后, 执行并一直沿用。但实际上 市场情况可能会发生变化, 例如 CTD券种类 及现券基点价值都会变动, 这些变化会使得 维持原有的套保比率达不到预期效果。因此 在实际应用中需要动态调整套保比率, 以使 套保效果得到改善。当利率或市场剧烈变动 时, 尤其需要动态套保。

\section{（4）套期效率}

本文采用Ederington提出的方法, 设置 指标比较套保前后的收益方差, 方差下降幅 度越大, 套保效果越好。套期效率评价指标 如下:

$$
H_{e}=\left[\operatorname{Var}\left(U_{t}\right)-\operatorname{Var}\left(H_{t}\right)\right] / \operatorname{Var}\left(U_{t}\right)
$$

\section{2 样本数据}

样本区间选取2014年10月8日至2015年 1月8日, 建仓时点为2014年11月25日。本文 选取2014年12月主力合约TF1503为套保期 货合约。另外选取多品种、多期限的债券作 为被套保现货债券, 比较其套保绩效。

首先需要确定TF1503在2014年11月25 日的CTD券。2014年11月25日, IRR最大 (基 差最小）的14附息国债24是TF1503合约的 CTD券, 并且交易活跃, 用它的收盘价计算
的隐含回购利率能够有效反应当前的利率 水平。因此选择140024为CTD券。

\section{3. 实证结果与分析}

\section{1 静态套期保值}

首先采用不同静态套保方法来确定国债期

\begin{tabular}{|c|c|c|c|c|}
\hline & 债券名称 & $\begin{array}{c}\text { HR } \\
(\mathrm{MD})\end{array}$ & $\begin{array}{c}\mathrm{HR} \\
(\mathrm{BPV})\end{array}$ & $\begin{array}{l}\text { HR } \\
(\beta)\end{array}$ \\
\hline \multirow{3}{*}{$\begin{array}{l}\text { 可交 } \\
\text { 割券 }\end{array}$} & 130015.IB & 0.8556 & 0.8535 & 0.6865 \\
\hline & 110015.IB & 1.0137 & 1.0112 & 0.9868 \\
\hline & 140024.IB & 1.0437 & 1.0411 & 1.0411 \\
\hline \multirow{3}{*}{$\begin{array}{c}\text { 短国 } \\
\text { 债 }\end{array}$} & 140014.IB & 0.1061 & 0.1059 & 0.1037 \\
\hline & 130017.IB & 0.2858 & 0.2851 & 0.2903 \\
\hline & 140020.IB & 0.4571 & 0.4559 & 0.3731 \\
\hline \multirow{3}{*}{$\begin{array}{c}\text { 长国 } \\
\text { 债 }\end{array}$} & 130005.IB & 1.2166 & 1.2136 & 1.1468 \\
\hline & 140012.IB & 1.4121 & 1.4086 & 1.2691 \\
\hline & 140021.IB & 1.4521 & 1.4485 & 1.2913 \\
\hline \multirow{3}{*}{$\begin{array}{c}\text { 中 } \\
\text { 金融 } \\
\text { 债 }\end{array}$} & 140445.IB & 0.7624 & 0.7605 & 1.0451 \\
\hline & 130246.IB & 0.9189 & 0.9166 & 1.4451 \\
\hline & 140203.IB & 0.9811 & 0.9787 & 1.5625 \\
\hline \multirow{3}{*}{$\begin{array}{c}\text { 长 } \\
\text { 金融 } \\
\text { 债 }\end{array}$} & 130231.IB & 1.2471 & 1.2440 & 1.8181 \\
\hline & 140205.IB & 1.4266 & 1.4230 & 2.3896 \\
\hline & 140222.IB & 1.4440 & 1.4404 & 2.4049 \\
\hline \multirow{3}{*}{$\begin{array}{c}\text { 短 } \\
\text { 金融 } \\
\text { 债 }\end{array}$} & 140443.IB & 0.1248 & 0.1245 & 0.1033 \\
\hline & 140311.IB & 0.2122 & 0.2117 & 0.2219 \\
\hline & 140201.IB & 0.3566 & 0.3557 & 0.4596 \\
\hline \multirow{3}{*}{$\begin{array}{l}\text { 短 } \\
\text { 融 }\end{array}$} & 041461042.IB & 0.1080 & 0.1077 & 0.0616 \\
\hline & 041452040.IB & 0.1245 & 0.1242 & 0.1437 \\
\hline & 041460082.IB & 0.1276 & 0.1272 & 0.1238 \\
\hline \multirow{3}{*}{$\begin{array}{l}\text { 中 } \\
\text { 票 }\end{array}$} & 1282156.IB & 0.3966 & 0.3956 & 0.4311 \\
\hline & 101363002.IB & 0.5963 & 0.5948 & 0.7192 \\
\hline & 101451017.IB & 0.7273 & 0.7255 & 0.8973 \\
\hline \multirow{3}{*}{$\begin{array}{c}\text { 企 } \\
\text { 业 } \\
\text { 债 }\end{array}$} & 1080034.IB & 0.6802 & 0.6785 & 0.1259 \\
\hline & 1080181.IB & 0.8254 & 0.8233 & 0.6527 \\
\hline & 1480341.IB & 0.7003 & 0.6985 & 0.6130 \\
\hline
\end{tabular}
货对多类债券最优套期率。债券品种的选择 包括国债: 可交割国债、不可交割的长短期 国债; 金融债：短期、中期、长期金融债; 信用债：短融、中票、企业债。对各债券的 最优套期率结果见表1。

表1 债券最优套期率 
在估计得到不同被套保债券的3种套 期保值比率之后, 对国债期货的套期保值效 果进行度量, 计算得到的套保有效性见表2。

表2 套保绩效检验

\begin{tabular}{|c|c|c|c|c|}
\hline & 债券名称 & $\begin{array}{c}\mathrm{He} \\
(\mathrm{MD})\end{array}$ & $\begin{array}{c}\mathrm{He} \\
\text { (BVP) }\end{array}$ & $\begin{array}{l}\mathrm{He} \\
(\beta)\end{array}$ \\
\hline \multirow{3}{*}{$\begin{array}{l}\text { 可交 } \\
\text { 割券 }\end{array}$} & 130015.IB & $44.64 \%$ & $44.94 \%$ & $62.04 \%$ \\
\hline & 110015.IB & $58.32 \%$ & $58.59 \%$ & $61.11 \%$ \\
\hline & 140024.IB & $57.83 \%$ & $58.08 \%$ & $58.08 \%$ \\
\hline \multirow{3}{*}{$\begin{array}{c}\text { 短国 } \\
\text { 债 }\end{array}$} & 140014.IB & $-86.97 \%$ & $-86.49 \%$ & $-82.56 \%$ \\
\hline & 130017.IB & $-81.99 \%$ & $-80.36 \%$ & $-77.06 \%$ \\
\hline & 140020.IB & $-84.84 \%$ & $-84.10 \%$ & $-37.40 \%$ \\
\hline \multirow{3}{*}{$\begin{array}{c}\text { 长国 } \\
\text { 债 }\end{array}$} & 130005.IB & $47.04 \%$ & $47.32 \%$ & $53.13 \%$ \\
\hline & 140012.IB & $19.10 \%$ & $19.53 \%$ & $34.60 \%$ \\
\hline & 140021.IB & $12.36 \%$ & $12.82 \%$ & $30.36 \%$ \\
\hline 中 & 140445.IB & $-11.54 \%$ & $-11.35 \%$ & $-48.44 \%$ \\
\hline \multirow{2}{*}{$\begin{array}{c}\text { 金融 } \\
\text { 债 }\end{array}$} & 130246.IB & $37.65 \%$ & $37.75 \%$ & $-11.03 \%$ \\
\hline & 140203.IB & $37.78 \%$ & $37.90 \%$ & $-19.61 \%$ \\
\hline 长 & 130231.IB & $30.14 \%$ & $30.22 \%$ & $2.32 \%$ \\
\hline \multirow{2}{*}{$\begin{array}{c}\text { 金融 } \\
\text { 债 }\end{array}$} & 140205.IB & $29.20 \%$ & $29.28 \%$ & $-20.36 \%$ \\
\hline & 140222.IB & $25.45 \%$ & $25.54 \%$ & $-25.74 \%$ \\
\hline 短 & 140443.IB & $-5.69 \%$ & $-5.59 \%$ & $0.00 \%$ \\
\hline \multirow{3}{*}{$\begin{array}{c}\text { 金融 } \\
\text { 债 }\end{array}$} & 140311.IB & $-14.03 \%$ & $-13.86 \%$ & $-17.26 \%$ \\
\hline & 140201.IB & $42.44 \%$ & $42.52 \%$ & $27.02 \%$ \\
\hline & 041461042.IB & $2.15 \%$ & $2.20 \%$ & $6.29 \%$ \\
\hline \multirow[t]{2}{*}{ 短融 } & 041452040.IB & $1.60 \%$ & $1.64 \%$ & $-1.10 \%$ \\
\hline & 041460082.IB & $-10.61 \%$ & $-10.54 \%$ & $-9.81 \%$ \\
\hline \multirow{3}{*}{ 中票 } & 1282156.IB & $-25.49 \%$ & $-25.29 \%$ & $-32.64 \%$ \\
\hline & 101363002.IB & $-11.17 \%$ & $-11.01 \%$ & $-26.77 \%$ \\
\hline & 101451017.IB & $-12.44 \%$ & $-12.33 \%$ & $-23.51 \%$ \\
\hline \multirow{3}{*}{$\begin{array}{c}\text { 企业 } \\
\text { 债 }\end{array}$} & 1080034.IB & $-6.88 \%$ & $-6.80 \%$ & $2.73 \%$ \\
\hline & 1080181.IB & $-6.94 \%$ & $-6.84 \%$ & $4.88 \%$ \\
\hline & 1480341.IB & $-12.63 \%$ & $-12.52 \%$ & $-1.94 \%$ \\
\hline
\end{tabular}

从套期保值绩效可以发现, 并非所有券 种都适用于国债期货的套期保值。套保效果 与价格相关系数、期限长短, 风险性质都有 紧密关系。可交割债券跟国债期货的价格保 持非常高度的相关性, 其中和CTD券的相关 性最高, 对最便宜可交割债券的套保绩效也 最佳。例如国债期货在2014年11月25的CTD 券140024和多次担任CTD券的110015都能 对冲掉接近 $60 \%$ 的收益率方差。
另外，剩余期限为中长期的国债、金融 债和可交割债券套期保值效果也比较理想, 能平均对冲 $28 \%$ 的收益方差。这是因为他们 跟国债期货的期限结构相同, 收益率受到共 同因素的影响，因此国债期货可以很好的对 冲风险。但是期限较短的国债和金融债的套 保效果不好, 某些收益率方差甚至在套保期 间有所上升，因为这类债券价格更多受到短 期因素，比如说资金面等影响较大，与国债 期货的期限相差大，因此套保效果欠佳。

信用债的套保效果大多不好, 由于以下 几点原因所造成。第一，信用债有较大的信 用风险, 投资者需要获得额外的补偿, 收益 率较高。由于国债期货不是信用衍生品, 无 法对冲信用风险。套保期间由于信用利差的 不断变化, 导致套保绩效不佳。第二, 本文 套期保值样本期间, 信用债的波动率较大, 使得套保基差风险变大, 影响了套保绩效。

关于套保方法的选择，修正久期法和基 点价值法的套保绩效相差不大, 相比较而言 基点价值法的效果稍好。经过收益率 $\beta$ 调整 后的绩效有所提升。

\section{2 动态套期保值}

选取与前文一致的数据样本, 以 130015 为例进行动态调整套保, 国债期货仍选择 TF1503合约。考虑到实际情况选择每周进 行一次调整, 具体为 2014 年11月25日开始 进行为期一个半月的套期保值, 在每周五进 行套期保值比例的调整, 如果周五不是交易 日，则顺延到下一个周五再进行调整。

在每个套保比例调整的时刻, 需要重新 计算每个可交割债券的隐含回购利率, 选择 隐含回购利率最高的可交割债券, 即此时国 债期货所对应的CTD券。然后利用CTD券的 基点价值和转换因子来计算国债期货的基 点价值。动态调整的重要参数见表3。

表4则具体比较了动态套保与静态套保 的效率。表4可见动态套保法下收益率方差 的波动性进一步降低, 单降低幅度均小于 $3 \%$, 动态效果并不明显。可能的原因是样 本期间内CTD券基本稳定在 140024 , 套保比 例的变动幅度不大, 从而收益方差的进一步 降低效果不明显。从另一方面说, 频繁的进 
行调整会带来大量的交易成本, 需要综合考 虑调整成本。

表3 动态套保的参数调整

\begin{tabular}{cccccc}
\hline \multirow{2}{*}{ 日期 } & CTD券 & $\begin{array}{c}\text { CTD } \\
\text { 基点 } \\
\text { 价值 }\end{array}$ & $\begin{array}{c}\text { TF1503 } \\
\text { 基点价 } \\
\text { 值 }\end{array}$ & $\begin{array}{c}130015 \\
\text { 基点价 } \\
\text { 值 }\end{array}$ & $\begin{array}{c}\text { 套保 } \\
\text { 比例 }\end{array}$ \\
\hline $14 / 11 / 25$ & $140024 . I B$ & 0.061 & 585.333 & 0.050 & 0.853 \\
$14 / 12 / 2$ & $120004 . I B$ & 0.063 & 614.784 & 0.050 & 0.811 \\
$14 / 12 / 9$ & $130015 . I B$ & 0.049 & 478.346 & 0.049 & 1.022 \\
$14 / 12 / 16$ & $140024 . I B$ & 0.059 & 571.489 & 0.049 & 0.856 \\
$14 / 12 / 23$ & $140024 . I B$ & 0.060 & 574.895 & 0.049 & 0.853 \\
$14 / 12 / 30$ & $140024 . I B$ & 0.060 & 574.112 & 0.049 & 0.854 \\
$2015 / 1 / 6$ & $140013 . I B$ & 0.058 & 547.406 & 0.049 & 0.893 \\
\hline
\end{tabular}

表4 套保绩效比较

\begin{tabular}{lll}
\hline 130015 & 静态 $\mathrm{He}$ & 动态 $\mathrm{He}$ \\
\hline 基点价值法 & $44.94 \%$ & $47.81 \%$ \\
$\beta$ 调整 & $62.04 \%$ & $64.42 \%$ \\
\hline
\end{tabular}

\section{4. 结束语}

从国债期货套期保值的适用性来说, 被 套保债券与国债期货价格的相关性, 二者剩 余期限的差距以及被套保债券收益率的组 成都会对套保效果产生影响。实证结果可 见，国债期货对可交割债券、长期金融债、 以及对于剩余期限较长的国债均有不错的 套保效果，在一定程度上对冲其利率风险; 但是对剩余期限较短的国债和金融债套保 效果欠佳; 信用债由于存在信用风险, 也不 适合采用国债期货进行套期保值。关于国债 期货套期保值方法的选择上, 基点价值法和 久期法所得到的结果相差不大, 经过 $\beta$ 调整 后, 套保的绩效一般会上升。当套保期间 CTD券多次变化或基点价值变动剧烈时, 动 态套期保值将会有显著的绩效提升效果。

\section{References}

[1] Johnson L., The Theory of Hedging and Speculation in Commodity Futures, Review of Economic Studies,vol.27,pp.139-151, 1960.

[2] SteinJ L., The Simultaneous Determination of Spot and Futures Prices, American Economic Review,vol. 51,pp. 1012-1025, 1961.

[3] Ederington L H., The Hedging Performance of the New Futures Market, Journal of Finance, vol. 34, pp. 157-170, 1979.

[4] Witt H J and T C Schroeder., Comparison of analytical approaches for estimating hedge ratios for agricultural commodities,The Journal Futures Markets,vol. 7, pp. 135-146, 1987.

[5] Ghosh A., Hedging with Stock Index Futures: Estimation and Forecasting with Error Correction Model,The Journal Futures Markets, vol. 13, pp. 743-752, 1993.

[6] 卢太平和刘心报,股指期货套期保值理 论及模型的演进与实证研究, 经济研究 导刊, vol. 16, pp. 58-61, 2011.

[7] 程宇和吕广仁,利率风险管理中利率期 货套期保值研究, Proceedings of International Conference on Engineering and Business Management (EBM2010), pp. 3777-3780, 2010.

[8] 傅晓云. 国债期货在我国商业银行利率 风险管理中的应用研究，新金融,vol. 10, pp. 56-60, 2013. 